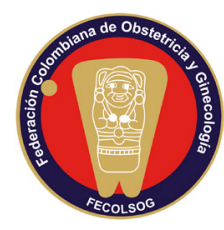

\title{
La seguridad en los servicios de salud, un problema prioritario en la atención de la mujer a nivel mundial
}

\author{
Hernando Gaitán-Duarte, MD, MSc. ${ }^{1}$; Kelly Estrada-Orozco, MD, MSc, PhD (c). ${ }^{2}$
}

a seguridad del paciente ha sido definida por la Organización Mundial de la Salud (OMS) como "la gestión de los procesos que conllevan a una reducción del riesgo de ocasionar un daño innecesario en la atención médica a un mínimo aceptable” (1, p.15). Como mínimo aceptable se entienden "aquellos daños que son inevitables bajo los conocimientos actuales, los recursos disponibles y el contexto en el que se presta la atención y que se contrasta con el riesgo de no recibir tratamiento o recibir otro tratamiento" (1, p.15). El daño al paciente es el resultado no deseado que ocurre más por la atención en salud que por la enfermedad de base (2), y se conoce como evento adverso o evento reportable con efectos no deseados -EREND- (3).

La Real Academia Española define al paciente como "la persona que padece física y corporalmente, y especialmente quien se halla bajo atención médica" o "persona que es o va a ser reconocida médicamente" (4). Este concepto incluye tanto al enfermo como al sujeto sano que está en contacto con los servicios de salud. Incluye a los sujetos con condiciones patológicas que reciben atención y cuidados durante la hospitalización, aspecto que ha sido ampliamente estudiado $(5,6)$, así mismo incluye a quienes reciben atención ambulatoria (7) y a personas con buena salud como los niños para control de crecimiento y desarrollo, o de las mujeres en control prenatal.

1. Editor Revista Colombiana deObstetriciay Ginecología (RCOG), Bogotá(Colombia)

2. Editora Invitada Facultad de Medicina, Universidad Nacional de Colombia, Bogotá (Colombia)
La gestante asiste al control prenatal para verificar el curso normal del embarazo, así mismo para poder detectar de forma oportuna posibles condiciones patológicas que pueden afectar la salud de ellas o del fruto de la gestación. Por ejemplo, las gestantes o el feto son susceptibles de enfermar por condiciones adquiridas antes o durante el embarazo, como la sífilis congénita (8), por situaciones propias del embarazo como la preeclampsia (9), o por complicaciones de los eventos fisiológicos durante la finalización del embarazo como la hemorragia postparto (10). Todas estas condiciones requieren el reconocimiento médico oportuno para reducir los riesgos a unos mínimos aceptables, tanto para la madre como para el feto. En el caso de la sífilis congénita el riesgo aceptable no debería ser superior al 5 x 10.000 según la meta de eliminación de la sífilis congénita (11); para las gestantes que desarrollan preeclampsia, el riesgo de morir por eclampsia no debería ser mayor al 2\% (12); y respecto a la hemorragia postparto, el riesgo de presentar morbilidad materna extrema por esta causa no debería ser mayor al 2,5\% y el riesgo de morir por esta causa no debería ser mayor al 2\% (13).

Estos datos considerados como riesgos mínimos actuales son tomados de los países de altos ingresos que podrían ser considerados como los mejores alcanzados hasta ahora. ¿Cómo explicar que el riesgo de estos malos resultados en salud sean mayores en los países de medios y bajos ingresos? En parte podría ser explicado por los determinantes de la salud (DS). Los DS son un conjunto de factores del individuo y de 
otras esferas como la social, económica y ambiental que determinan el estado de salud de los individuos o de las poblaciones. Se encuentran clasificados en dos grupos: 1) los que son responsabilidad multisectorial del Estado, son estructurales e incluyen determinantes políticos, económicos y sociales; y 2) los determinantes de la salud intermedios y proximales, que median directamente en los estados de salud y enfermedad. Estos últimos incluyen los estilos de vida, factores ambientales, aspectos biológicos y genéticos de la población y los relacionados con la atención sanitaria, que a su vez incluye las características del proceso de atención en salud (14), que reúnen características de los procesos administrativos, características del entorno, naturaleza del trabajo, características de los equipos e individuos que brindan la atención y las características de los pacientes (15).

Los servicios de salud a menudo fallan en el acceso, la calidad y suficiencia para algunos sectores de la población. Estas fallas afectan más a las mujeres, indígenas, migrantes, comunidades afro y grupos diversos que, además, sufren marginalización, discriminación y violación de derechos, tales como derecho a la educación, al agua potable, a los servicios sanitarios, a la alimentación y la salud (16). Las diferencias en relación con la salud y la atención entre poblaciones y entre las regiones se han descrito en la literatura durante más de 30 años y se presentan independientemente del sistema político o económico que prevalezca en esa región. Cuando estas diferencias son innecesarias, evitables, arbitrarias e injustas, se considera que existe inequidad en salud (17).

La inequidad en salud incrementa la mortalidad materna e infantil, es un factor diferenciador de los indicadores de salud entre los países, pero también en el interior de un país. La inequidad en salud relacionada con la etnia, por ejemplo, explica una proporción de la morbimortalidad materna e infantil en el mundo (18). En Colombia, este fenómeno ha sido descrito en la zona pacífica poblada mayoritariamente por comunidades afro e indígenas (19). También se han descrito otros factores que generan inequidad en salud, como el tipo de aseguramiento que se ha asociado a un incremento en el riesgo de malos resultados en las mujeres gestantes que reciben atención en el régimen de aseguramiento subsidiado por el Estado, frente a aquellas que reciben atención en el régimen contributivo (20).

Las diferencias en los resultados no deseados no son exclusivas de la población gestante, también de las pacientes que requieren atención por patología ginecológica, por ejemplo, por cáncer cervical, infecciones de transmisión sexual y problemas uro-ginecológicos que incluyen los problemas del piso pélvico, quienes son vulnerables a este tipo de inequidades (21). En un sentido más amplio, en el proceso de atención ocurren resultados no deseados no solo por las acciones asociadas a la atención, como es la inadecuada interpretación de una prueba diagnóstica no treponémica en la tamización de la sífilis gestacional, o por la omisión en la provisión de cuidado como puede ocurrir al no proporcionar en el servicio una prueba rápida para ser aplicada e interpretada en el sitio de atención; también ocurre por la falta de atención oportuna no solo a los sujetos con enfermedades, sino en quienes se busca prevenir las enfermedades o promocionar la salud. Esta omisión puede ocasionar daño no intencional al sujeto, como sucede cuando una mujer gestante no accede al control prenatal. En Colombia, el efecto de la deficiencia y las acciones de prevención son observables con los casos incidentes de sífilis congénita. Esta condición prevenible con tratamiento ofrecido a la madre durante la gestación tuvo una incidencia acumulada de 1,54 por cada 1000 nacidos vivos para 2018, y el promedio nacional en 2021 se ubica en 2,03 casos por 1000 nacidos vivos, sin embargo, existen claras diferencias entre las regiones; hay departamentos que superan el promedio nacional, como es el caso de Arauca y Casanare con 3,78 casos por mil nacidos vivos (22). Este problema de oportunidad en la atención es motivo en el presente número de la RCOG, de un reporte de casos de sífilis congénita en Bogotá. En este estudio se describen fallas en la cobertura o en la previsión de 
la atención a las gestantes que resultaron en daño al feto o al recién nacido.

Todos estos malos resultados, que finalmente son EREND, deberían ser objeto de programas de gestión del riesgo clínico como parte de la seguridad del paciente o de la seguridad en la atención en salud, por lo que es tarea de todos los actores del sector salud fortalecer las actividades que reduzcan el riesgo de aparición de daños en los sujetos que precisan de servicios de salud en todos los niveles de atención, ya sean actividades de promoción y prevención, atención primaria y la atención especializada, ambulatoria u hospitalaria. En esta misión de garantizar la seguridad en la atención en salud somos responsables los entes gubernamentales nacionales y locales, los terceros pagadores (conocidos como Entidades Administradoras de Planes de Beneficios de Salud -EAPB-), las instituciones hospitalarias, los centros de atención primaria e inclusive los centros comunitarios, ya sean privados o públicos.

Por esta razón, a partir de este número la RCOG abre una ventana para la publicación de investigaciones, primarias o secundarias, dirigidas a incrementar la seguridad en los servicios de salud relacionados con los EREND, derivados de la ausencia de los servicios de salud requeridos por la población, en nuestro caso la mujer, o como consecuencia de su interacción con la atención recibida en los servicios de salud. Buscamos aportar evidencia para reducir al mínimo los riesgo del daño por acción o por omisión en favor de la salud de la mujer en sus diferentes etapas de la vida, respecto a los métodos de vigilancia, las metodologías aplicadas para la gestión del riesgo y sus efectos en resultados de implementación, seguridad y efectividad, las implicaciones económicas y éticas de las intervenciones destinadas al incremento de la seguridad y las acciones destinadas a la reducción de las barreras para su adecuada implementación, aplicadas en los campos de la salud donde pueden beneficiar a la mujer. En el presente número iniciamos la difusión de estudios de investigación en seguridad en los servicios de salud con la publicación de la revisión sistemática de alcance sobre la estructura y funcionamiento de los equipos de respuesta rápida en entornos hospitalarios.

La intención es que con el tiempo la seguridad en la atención sea un objetivo común de trabajo en nuestro sistema de salud; no por el hecho de tener recursos limitados debemos aceptar los malos resultados, sino que debemos prevenirlos, reconocerlos y abordarlos desde un punto de vista sistémico para que el proceso de atención en todas sus dimensiones produzca mejores resultados en salud y disminuir las brechas de inequidad que actualmente se presentan en la población colombiana que, como se vio, afecta de manera especial a la mujer.

\section{REFERENCIAS}

1. World Health Organization. Conceptual Framework for the International Classification for Patient Safety. Version 1.1 Final Technical Report. January 2009. Disponible en: https://www.who.int/patientsafety/ taxonomy/icps_full_report.pdf

2. Vincent $\mathrm{C}$, Neale G, Woloshynowych M. Adverse events in British hospitals: preliminary retrospective record review. BMJ. 2001; 322(7285):517-9. https://doi. org/10.1136/bmj.322.7285.517

3. Estrada-Orozco K, Gaitán-Duarte H, Moreno S, Moreno Chaparro J. Reportable hospital events: incidence and contributing factors in the surgery service of a high complexity hospital in Bogotá, Colombia, 2017. Colombian Journal of Anesthesiology. 2019; 47(1):513. https://doi.org/10.1097/CJ9.0000000000000091

4. Real Academia de la lengua. Disponible en: https://dle. rae.es/paciente

5. Brennan TA, Leape LL, Laird NM, Hebert L, Localio AR, Lawthers AG, et al. Incidence of adverse events and negligence in hospitalized patients. Results of the Harvard Medical Practice Study I. N Engl J Med. 1991 7;324(6):370-6. https://doi.org/10.1056/ NEJM199102073240604

6. Aranaz-Andrés JM, Aibar-Remón C, Limón-Ramírez R, Amarilla A, Restrepo FR, Urroz O, et al. Prevalence ofadverse events in the hospitals of five Latin American countries: results of the 'Iberoamerican study of adverse 
events' (IBEAS). BMJ Qual Saf 2011; 20:1043-51. https://doi.org/10.1136/bmjqs.2011.051284

7. Montserrat-Capella D, Suárez M, Ortiz L, Mira JJ, Duarte HG, Reveiz L, et al. Frequency of ambulatory care adverse events in Latin American countries: the AMBEAS/PAHO cohort study. Int J Qual Health Care. 2015; 27(1):52-9. https://doi.org/10.1093/intqhc/mzu100

8. Fiumara N, Fleming W, Dowing JG, Good FL. The incidence of prenatal syphilis at the Boston City Hospital.N Engl J Med1952;247:48-52. https://doi. org/10.1056/NEJM195207102470203

9. Leeman L, Fontaine P. Hypertensive disorders of pregnancy. Am Fam Physician 2008; 78:93-100.

10. Committee on Practice Bulletins-Obstetrics. Practice Bulletin No. 183: Postpartum Hemorrhage. Obstet Gynecol 2017;130: e168-86. https://doi.org/10.1097/ AOG.0000000000002351

11. World Health Organization. The global elimination of congenital syphilis: rationale and strategy for action. 2007. 38 p. https://apps.who.int/iris/handle/10665/43782.

12. Ghulmiyyah L, Sibai B. Maternal mortality from preeclampsia/eclampsia. Semin Perinatol. 2012;36(1):56-9. https://doi.org/10.1053/j. semperi.2011.09.011

13. Maswime S, Buchmann E. A systematic review of maternal near miss and mortality due to postpartum hemorrhage. Int J Gynaecol Obstet. https://doi. org/10.1002/ijgo.12096

14. Villar Aguirre M. Factores determinantes de la salud: Importancia de la prevención. Acta Médica Peruana. 2011; 28:237-41.

15. Henriksen K, Elizabeth D, Keyes MA, Carayon P, Hughes R. Understanding Adverse Events: A Human Factors Framework. Rockville (MD): Agency for Healthcare Research and Quality (US): Agency for Healthcare Research and Quality (US); 2008. Disponible en https://www.ncbi.nlm.nih.gov/books/ NBK2666/

16. United Nations. UN75 2020 and beyond. Shaping our future. Disponible en: https://www.un.org/en/un75/ inequality-bridging-divide
17. Whitehead M. Los Conceptos y Principios de la Equidad en la Salud Washington: Organización Mundial de la Salud; 1991.

18. Small MJ, Allen TK, Brown HL. Global disparities in maternal morbidity and mortality. Semin Perinatol. 2017; 41(5):318-322. https://doi.org/10.1053/j. semperi.2017.04.009

19. Sandoval-Vargas YG, Eslava-Schmalbach JH. Inequidades en mortalidad materna por departamentos en Colombia para los años (2000-2001), (2005-2006) y (2008-2009) [Inequality regarding maternal mortality in Colombian departments in 2000-2001, 20052006 and 2008-2009]. Rev Salud Pública (Bogotá). 2013; 15(4):529-41. https://doi.org/10.1016/j. jval.2012.03.1106

20. Rivillas JC, Devia-Rodríguez R, Ingabire MG. Measuring socioeconomic and health financing inequality in maternal mortality in Colombia: a mixed methods approach. Int J Equity Health. 2020; 19(1):98. https:// doi.org/10.1186/s12939-020-01219-y

21. Robinson N, Stoffel C, Haider S. Global women's health is more than maternal health: a review of gynecology care needs in low-resource settings. Obstet Gynecol Surv. 2015;70(3):211-22. https://doi. org/10.1097/OGX.0000000000000166

22. Incidencia de Sífilis Congénita - Georeferenciado. 2020. Disponible en: https:/www.asivamosensalud. org/indicadores/gestion-del-riesgo/incidencia-desifilis-congenita-georeferenciado. 